Research Paper

\title{
Deregulation of CSMD1 targeted by microRNA-10b drives gastric cancer progression through the NF-KB pathway
}

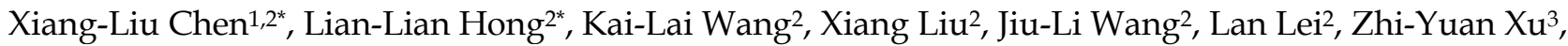
Xiang-Dong Cheng 3 and Zhi-Qiang Ling1,2 ${ }^{\bowtie}$

1. Department of Digestive Oncology, the First Affiliated Hospital of Wenzhou Medical University; the First Provincial Wenzhou Hospital of Zhejiang, Wenzhou 325000

2. Zhejiang Cancer Institute, Institute of Cancer Research and Basic Medical Sciences of Chinese Academy of Sciences, Cancer Hospital of University of Chinese Academy of Sciences, Zhejiang Cancer Hospital, Hangzhou 310022, China.

3. Department of Digestive Oncology, Zhejiang Province Cancer Hospital, Zhejiang Cancer Center, Hangzhou 310022, China.

*Contributed equally to this work.

$\square$ Corresponding author: Professor, Zhi-Qiang Ling, M.D., PhD. No.1 Banshan East Rd., Gongshu District, Hangzhou 310022, China. Telephone: +86-571-88122423; Fax: +86-571-88122423; Email: lingzq@zjcc.org.cn; MSN: lingzq@hotmail.com

(c) The author(s). This is an open access article distributed under the terms of the Creative Commons Attribution License (https://creativecommons.org/licenses/by/4.0/). See http://ivyspring.com/terms for full terms and conditions.

Received: 2017.11.11; Accepted: 2019.06.05; Published: 2019.08.06

\begin{abstract}
Aim: This study aimed to investigate the oncogenic activity of microRNA-10b by targeting CUB and sushi multiple domains protein 1 (CSMDI) in human gastric cancer (GC) and the underlying mechanisms.

Methods: The expression of CSMDI in human GC tissues was evaluated by real-time reverse transcription polymerase chain reaction (RT-PCR), immunoblotting, and immunohistochemical analysis. The expressive abundance of microRNA-10b was detected by stem-loop RT-PCR. Molecular and cellular techniques, including lentiviral vector-mediated knockdown or overexpression, were used to elucidate the effect of microRNA-10b on the expression of CSMD1.

Results: CSMD1 was targeted and downregulated by microRNA-10b in human GC tissues and cells, and the down-regulated expression of CSMDI contributed to poor survival. The knockdown of microRNA-10b expression inhibited cell proliferation in GC cells in vitro and tumor growth in vivo. The inhibition of microRNA-10b expression repressed invasion and migration of HGC27 cells and retarded GC cells metastasis to the liver in Balb/c nude mice. The up-regulated expression of microRNA-10b promoted the proliferation and metastasis of MKN74 cell in vitro. Intratumoral injection of microRNA-10b mimic also promoted the growth and metastasis of tumor xenografts in Balb/c nude mice. Mechanistically, microRNA-10b promoted the invasion and metastasis of human GC cells through inhibiting the expression of CSMDI, leading to the activation of the nuclear factor-KB (NF-KB) pathway that links inflammation to carcinogenesis, subsequently resulting in the upregulation of c-Myc, cyclin DI (CCNDI), and epithelial-mesenchymal transition (EMT) markers.

Conclusions: The findings established that microRNA-10b is an oncomiR that drives metastasis. Moreover, a set of critical tumor suppressor mechanisms was defined that microRNA-10b overcame to drive human GC progression.
\end{abstract}

Key words: microRNA-10b; CUB and sushi multiple domains protein 1 (CSMD1); gastric carcinoma (GC); epithelial-mesenchymal transition (EMT); Tumor metastasis.

\section{Introduction}

Gastric cancer (GC) is the fifth most common tumor and the third deadliest from cancer worldwide, making up $7 \%$ of cases and $9 \%$ of deaths [1]. It is particularly prevalent in East Asia, such as Japan, Korea, China, and so on [2]. Despite a steadily declining incidence, it newly occurred in 950,000 
people and caused 723,000 deaths in 2012 [1]. In the past few decades, although the diagnosis and treatment of gastric cancer has indeed improved significantly, the 5-year survival rate of patients with advanced gastric cancer, especially metastatic gastric cancer, is still very low [3]. Hunman CUB and sushi multiple domains protein 1 (CSMD1) is a novel candidate tumor suppressor gene located on the $p$ arm of chromosome 8 (8p23) [4]. Previous studies have shown that the deletion of $8 \mathrm{p} 23.2$ or reduced expression of CSMD1 has something to do with poor prognosis in many cancers [5-9]. Multiple mechanisms can reduce the expression of CSMD1. The allelic loss, copy number aberrations, mutations, and methylations of CSMD1 have been detected in various malignant tumours such as head and neck tumor, colorectal cancers, liver cancer, and so on [5-16]. Besides, it has been proved that the change of microRNA expression and dysfunction play an important role in tumorigenesis and metastasis by regulating target genes and pathways, subsequently resulting in the alteration of proliferation, differentiation, apoptosis, invasion and metastasis of tumor cells [17-19]. Therefore, the molecular mechanism underlying the microRNAs-driven deregulation of CSMD1 contributing to GC metastases need to be urgently unraveled.

MicroRNA is a small single-stranded non-coding RNA, that is a post-transcriptional negative regulator that binds completely or partly to the complementary sites in the 3'-untranslated region (3'UTR) of target mRNAs [17-19]. Accumulating evidence has shown that miRNAs can modulate tumor growth, metastasis, and progression by regulating multiple target genes [20-22]. microRNA-10b has been found to be highly expressed in many malignant tumours and related to the progress of breast cancer [23], colorectal cancer [24], head and neck cancer [25], pancreatic adenocarcinoma [26], glioblastoma [27], nasopharyngeal cancer [28], and liver cancer [29]. The pleiotropic nature of microRNA-10b was due to its suppression of multiple tumor suppressors, including ras homolog family member $\mathrm{C}$ (RhoC), urokinase plasminogen activator receptor (uPAR), matrix metalloproteinases (MMPs), tumor protein p53 (TP53), forkhead box O3 (FOXO3), CYLD lysine 63 deubiquitinase (CYLD), paired box 6 (PAX6), patched 1 (PTCH1), homeobox D10 (HOXD10), notch receptor 1 (NOTCH1), BCL6 transcription repressor (Bcl-6), and Kruppel like factor 4 (KLF4) [29-33]. A recent study reported that microRNA-10b mediated transforming growth factor- $\beta 1$-regulated glioblastoma proliferation, migration, and epithelial-mesenchymal transition (EMT) [34].
Moreover, the up-regulation of microRNA- $10 \mathrm{~b}$ is closely related to the survival of patients and is a predictors of poor prognosis in some cancer patients [35-37]. This suggested that microRNA-10b could play a critical role in many types of human cancers.

Whether microRNA-10b acts as oncogene or tumor suppressor in GC is still not known. Kim et al. [38] reported that the expression of microRNA-10b was frequently silenced in GCs by its promoter methylation, and also microRNA-10b might act as a tumor suppressor by suppressing oncogenic microtubule associated protein RP/EB family member 1 (MAPRE1) at the transcriptional level, which was further confirmed by $\mathrm{Li}$ et al [39]. However, Liu et al. reported that microRNA-10b stimulates the up-regulated expression of RhoC and AKT serine / threonine kinase (AKT) phosphorylation by targeting HOXD10, thus promoting the invasion of gastric tumors [40]. Wang et al. also found that the expression of microRNA-10b was significantly up-regulated in GCs, which was related to tumor size, Lauren classification, invasive depth, lymphatic metastasis, distant metastasis, tumor- lymph node-metastasis (TNM) stage, and poor prognosis [41]. In this study, we found that the expression of microRNA-10b was up-regulated in gastric cancer, which was positively correlated with lymph node metastasis. The overexpression of $\mathrm{miR}-10 \mathrm{~b}$ promoted the proliferation and invasion of GC cells in vitro and the growth and liver metastasis of GC cells in nude mice. On the contrary, knockdown of microRNA-10b expression significantly inhibited the proliferation and invasion in vitro, and tumor growth of GC cells in vivo. The study concluded that miR-10b played an oncogenic role in GC metastasis.

\section{Material and methods}

\section{Cell lines, clinical tissue specimens of GC}

Six human GC cell lines (MKN74, AGS, BGC823, MKN28, SGC7901, HGC27) and a human fetal gastric mucosa epithelium cell line (GES-1) were purchased from the Cell Bank of the Chinese Academy of Sciences (Shanghai, P. R. China). Cells were grown in the Roswell Park Memorial Institute (RPMI)-1640 medium containing $10 \%$ fetal heat-inactivated bovine serum (FBS) $100 \mathrm{U} / \mathrm{mL}$ penicillin and $100 \mathrm{U} / \mathrm{mL}$ streptomycin (Gibco, Invitrogen Inc., CA, USA), maintained at $37^{\circ} \mathrm{C}$ incubator in a humidified atmosphere consisting of $5 \% \mathrm{CO}_{2}$.

A total of 62 GC patients [mean age, 55.5 years; range, $38-72$ years; $36(58.1 \%)$ male and $26(41.9 \%)$ female] who underwent radical resection of GC, without any preoperative treatment, at Institute of cancer research and basic medical sciences of Chinese 
Academy of Sciences, Cancer Hospital of University of Chinese Academy of Sciences, Zhejiang Cancer Hospital (No.1 Banshan East Road, Gongshu District, Hangzhou 310022, P. R. China.) from January 2011 to December 2012 were recruited in present study. All patients underwent pathological diagnosis by two professional pathologists independently according to the criteria of the American Joint Committee on Cancer (AJCC) / Union for International Cancer Control (UICC) TNM classification. Moreover, in 18 $(29.0 \%), 31(50.0 \%)$, and $13(21.0 \%)$ patients, the tumors were found in gastric antrum, gastric body, and gastric cardia, respectively. Further, 14 (22.6\%) patients had stage I/II GC and 48 (77.4\%) had stage III/IV GC. Based on the histological findings, 47 (75.8\%) patients had an intestinal type and 15 (24.2\%) had a gastric type cancer. The carcinoma was highly to moderately differentiated in $13(21.0 \%)$ patients and poorly differentiated in $49(79.0 \%)$ patients. None of the patients had any history of other tumors. Tumors and their paired paracancerous normal tissues were collected during surgery. The tissue specimens of this study were obtained with informed consent and were studied in accordance with the guidelines approved by Zhejiang Cancer Hospital Medical Ethics Committee (Hangzhou, China). The regular follow-up of all enrolled GC patients was performed by the special person until the end date of the project.

\section{Real-time reverse transcription polymerase chain reaction (RT-PCR)}

One-step total RNA extraction TRIzol reagent (Invitrogen, Carlsbad, California, USA) was used for the extraction of total cellular RNA from the cultured cells or frozen tissues. Reverse transcription of mRNA was performed using the PrimeScript RT reagent Kit (Takara, Otsu, Japan) as described by the manufacture. For reverse transcription (RT) reactions, stem-loop primers were used for cDNA synthesis in microRNA-10b experiments. Then the quantitative polymerase chain reaction (PCR) was carried out with primers for the expression abundance detection of microRNA-10b, CSMD1, E-cadherin, Twist, Snail, Zeb1, Vimentin, NFkB-p65, NFkB-p50, nitric oxide synthase 2 (iNOS), CCND1, c-MYC, glyceraldehyde-3- phosphate dehydrogenase (GAPDH), and U6. Quantitative RT-PCR reaction in ABI 7500 fast RT-PCR system (ABI, CA, USA) was carried out using SYBR Green PCR Master Mix (Invitrogen, Carlsbad, California, USA), according to the standard quantitative PCR program. The primer sequences used are listed in Table S1. The conditions of PCR amplification were as follows: $95^{\circ} \mathrm{C} 15$ minutes, then 40 cycles of $94^{\circ} \mathrm{C} 15$ seconds, $60^{\circ} \mathrm{C} 30$ seconds, and $70^{\circ} \mathrm{C} 35$ seconds. The comparative cyclic threshold method was used to calculate the relative expression and normalize it to GAPDH or U6 small nuclear (sn) RNA. All experiments were performed in triplicate, and the median values were taken.

\section{Immunohistochemical analysis}

Immunohistochemistry was performed as described in a previous study [42]. After deparaffinization, the antigen was recovered in 0.01 $\mathrm{M}$ citric acid buffer, and then the activity of endogenous peroxidase was inactivated in $3 \% \mathrm{H}_{2} \mathrm{O}_{2}$ in methanol for 10 mins. Nonspecific binding was blocked by incubation with $10 \%$ normal goat serum in phosphate-buffered saline (PBS) for $1 \mathrm{~h}$ at room temperature. Prior to the addition of an primary antibody, $10 \%$ of normal goat serum was incubated at room temperature in phosphate buffer (PBS) for $1 \mathrm{~h}$ to block non-specific binding reactions. The slides were incubated with primary antibodies against CSMD1 (Santa Cruz Biotechnology, Inc.2145 Delaware Ave Santa Cruz, CA. 95060, USA) overnight at $4^{\circ} \mathrm{C}$, and then added biotinylated goat anti-mouse immunoglobulin G (Sigma, MO, USA) for 1 hour at room temperature. Then, a streptavidin-biotin-peroxidase complex assay was performed. The peroxidase activity was developed by incubating with $0.1 \%$ 3,3-diaminobenzidine (Sigma) in PBS with $0.05 \% \quad \mathrm{H}_{2} \mathrm{O}_{2}$ for $5 \mathrm{~min}$ at room temperature. As described methods in our previous studies [42, 43], based on the frequency and intensity of staining, the scores of histochemical staining were determined by three independent clinical pathologists, and the inconsistent results were finally determined after discussion.

\section{Western blotting analysis}

The western blotting analysis was carried out using Immobilon-P polyvinylidene difluoride membranes (Millipore, 28820 Single Oak Drive, Temecula, California 92590, USA) as described in previous studies [42,43]. The cell lysates were separated on sodium dodecyl sulfate-polyacrylamide gels and the western blotting analysis was carried out with following antibodies against CSMD1, Cyclin D1 (Santa Cruz Biotechnology), Snail (Abgent Inc, CA, USA), E-cadherin, Vimentin, Twist, Zeb1 (Cell Signaling Technology, MA, USA), iNOS, NFkB-p65, NFkB-p50 (Abcam, Cambridge, CB4 0FL, UK), and c-MYC (Sigma Chemical Co., MO, USA), respectively. $\beta$-actin (TaKaRa Co. Ltd, Kusachi, Japan) was used as an internal control. The density of band was measured using the ChemiDoc ${ }^{\mathrm{TM}} \mathrm{XRS}^{+}$System (1000 Alfred Nobel Drive, Hercules, California 94547, USA) equipping with Image-Pro Plus software and Epson color image scanner. The data was normalized to the 
$\beta$-actin.

\section{Plasmid construction}

In order to study the overexpression of microRNA-10b, the primer pairs were designed to amplify a genome fragment of human microRNA-10b precursor using PCR method as described in a previous study [29]. The PCR product was cloned into pcDNA3.1 (Gibco, Invitrogen Inc., CA, USA) and named as pcDNA3.1-microRNA-10b. The negative control of Hsa-microRNA-10b inhibitor and inhibitor came from GenePharma Company (Shanghai, China). The pmiR-RB-REPORT luciferase vector (GenePharma, Shanghai, China) was used to construct the pMIRCSMD1-3'-UTP - wild-type (WT) or pMIR-CSMD1-3'-UTP-mut vectors. WT and mutant inserts were confirmed by sequencing. The pSilencer-nuclear factor $\mathrm{kB}$ (NFkB)-p65-shRNA recombinant plasmid vector, containing short hairpin RNA (shRNA) interfering sequence aiming at the target of NFkB-p65 gene, was constructed. The NFkB-p65-shRNA sequence was constructed as follows: 5'-GATCCgccetatccetttacgtcaTTCAAGAGAT GACGTAAAGGGATAGGGCtttttggaaa-3'; the lowercase part represents an interference sequence and the italic part represents a stem ring structure with the viscous ends of restriction endonuclease BamHI and HindIII at both ends. The constructed recombinant vector pSilencer-NFkB-p65-shRNA was sequenced, and the identified recombinant vector and negative control pSilencer were transfected into human GC cell line HGC27 by liposome transfection to verify its interference effect on NFkB-p65 gene, respectively. In the subsequent experiments, pSilencer-Negative Control (NC)-shRNA transfection was used as a negative control. The experiment was divided into three groups: pSilencer-NFkB-p65-shRNA transfection group, pSilencer-NC-shRNA transfection group (negative control group), and blank control group (only adding transfection reagent). The experiment was repeated three times.

\section{Cell transfection}

The cultured cells of $1 \times 10^{5}$ were plated to $50 \%$ confluence and transfected with the expression vector of microRNA-10b or the inhibitor lentiviral of hsa-microRNA-10b by Lipofectamine 2000 (Gibco, Invitrogen Inc., CA, USA) as described in previous studies [42]. The inhibitor of microRNA-10b was chemically enhanced with 2'-O-4'-C-methylene modification. The expression vector of microRNA-10b and the inhibitors of microRNA-10b were designed and synthesized by a professional biology co., Ltd (GenePharma Inc. Shanghai, China). The sequence thus obtained was as described in a previous study [44]. The transfection efficiency was detected by fluorescence image 24 hours later, and all kinds of experiments were carried out 48 hours later, and all the experiments were done in triplicate.

\section{Luciferase reporter assay}

The firefly luciferase reporter gene constructs and miR-10b-expressing plasmid were transiently transfected into cells with $80 \%$ confluence in 24-well plates. After 48 hours, the luciferase activity was determined by Dual-Luciferase Reporter Assay kit (Promega, Madison, WI, USA) as described in Kit's instructions, and the luciferase activity was normalized to Renilla luciferase activity.

\section{Cell growth assay}

For the 3-(4,5-Dimethylthiazol-2-yl)-2,5-diphenyltetrazolium bromide (MTT) assay, 1000 cells were inoculated in 96 well plate and transfected. The absorbance at $490 \mathrm{~nm}$ was measured once a day for 7 days. The soft agar assay is as follows: about 3000 cells were seeded in a 6-well plate and transfected. The size and number of soft agar colonies were detected after 14 days of transfection. All experiments were performed in triplicate, and the median values were taken.

\section{Migration assays}

The cells $\left(5 \times 10^{4}\right)$ were seeded into chambers

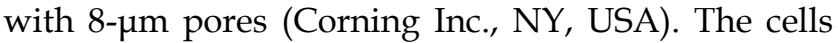
migrated to the bottom chamber through the membrane were fixed and stained with crystal violet after 24 hours. The numbers of migrating cells were counted by randomly selecting nine fields of view.

\section{Invasion assays}

The cells were seeded in the 24-well BD BioCoat Matrigel Invasion Chamber (BD Biosciences Discovery Labware, MA, USA) at a density of $1 \times 10^{6}$ per well. The serum (10\%) was used as a chemoattractant. The membrane of the upper chamber was fixed and stained using a Diff-Quik reagent (Sysmex, Kobe, Japan) after 22 hours of incubation. The invaded cells were counted in four randomly selected sites per membrane.

\section{Tumor cell xenograft assay in nude mice}

Six-week-old female BALB/c nude mice (weighing 20-22 g; Zhejiang Laboratory Animal Center, Hangzhou, China) were used in this study. All animal experiments were carried out in accordance with the agreement approved by the Animal Care and Use Committee of our Institute. For tumor growth experiments, $5 \times 10^{6}$ cells were subcutaneously injected the lower back regions of 6-week-old female nude 
mice for 4 consecutive weeks, and 3 nude mice in each group. The tumor growth was measured by caliper every other day, and the tumor volume was calculated using the formula as: volume $=$ length $\times$ width $^{2} \times 0.5$. Finally, the mice were sacrificed and tumor mass was harvested for examination.

\section{Statistical analysis}

All data were represented as mean \pm standard deviation (SD) of at least three independent experiments. The difference among groups was analyzed using the paired $t$ test for normal distribution by the $F$ test. All statistical analyses were performed using SPSS 19.0 (SPSS Inc., IL, USA). A $P$ value $<0.05$ was considered statistically significant. The survival curves and univariate analysis were carried out by the Kaplan-Meier method and log-rank test.
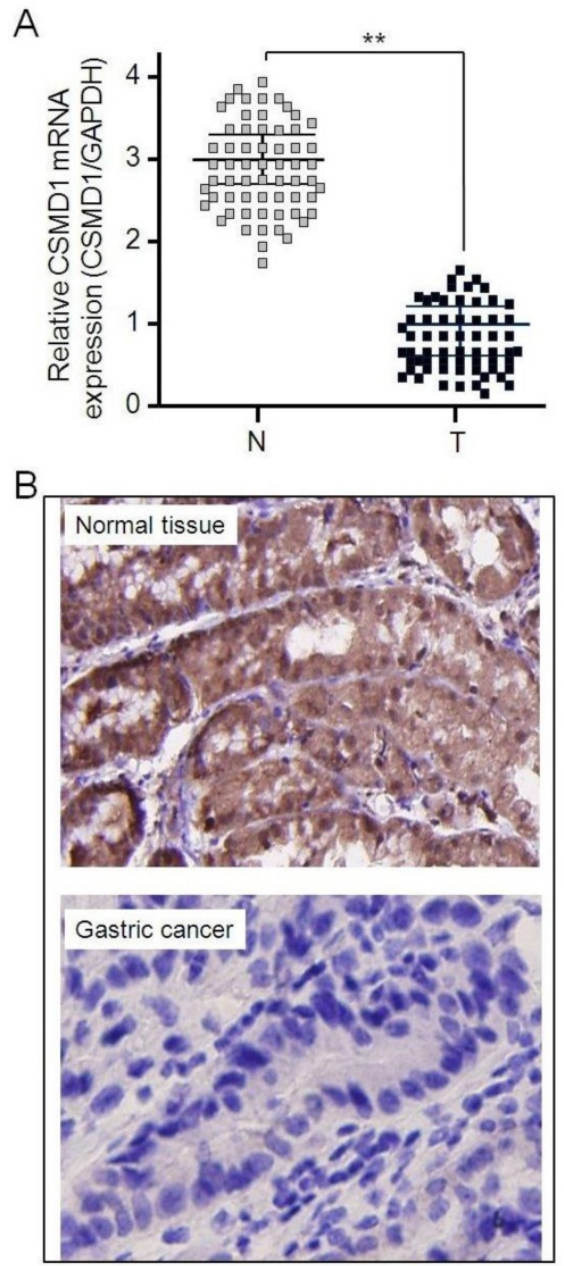

\section{Results}

\section{CSMDI was downregulated in GCs, which was} related to patients' prognosis

The expressive abundance of CSMD1 was determined in patients with GC by RT-PCR. CSMD1 was significantly downregulated in GC tissues compared with normal tissues (Figure 1A). This result was supported by the immunohistochemical staining (Figure $1 \mathrm{~B}$ and $1 \mathrm{C}$ ) and Western blot analysis (Figure 1D). Subsequently, the relationship between the expression of CSMD1 in GC tissues and their overall survival was investigated. The lower expression of CSMD1 in GC tissues was associated with the shorter survival time during 5-year follow-up $(P=0.016$, Kaplan-Meier survival and log-rank test) (Figure 1E), suggesting that the level of CSMD 1 in GC tissues was negatively correlated with patient's survival.

C

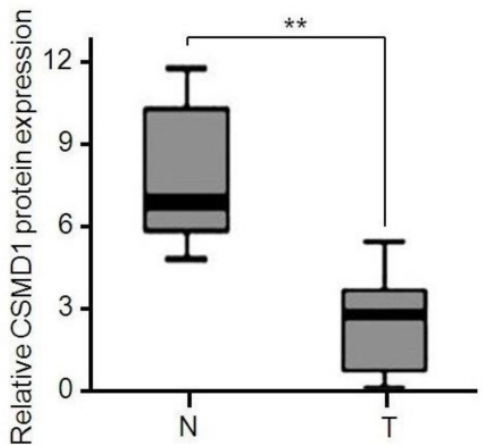

$\mathrm{D}$
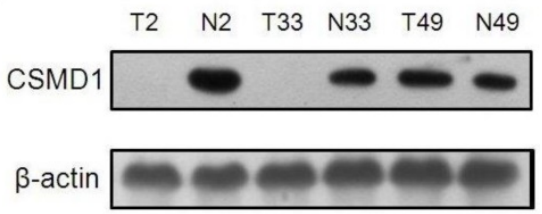

IHC score: $0 \quad 12 \quad 0 \quad 10 \quad 11 \quad 10$

$\mathrm{E}$

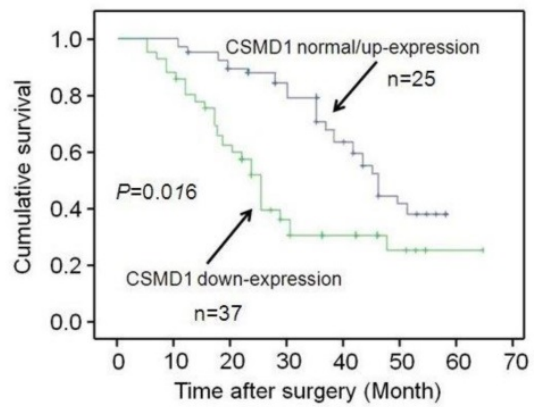

Figure 1. The expression of CSMD1 is downregulated in gastric tumor tissues and related to the survival of patients. (A) The relative levels of CSMD1 in sixty-two paired of GC samples were measured by real-time quantitative RT-PCR, and the GAPDH was used as an internal control. Student's test was used to analyze the significant differences between the GC and normal tissues, $* * p<0.01$. (B) The expression levels of CSMD1 were detected by immunochemistry analysis in 62 pairs of gastric tumor and matched adjacent normal tissues. Representative photos were shown in paired normal and tumor tissues $(\times 200)$. (C) The comparison of staining scores of CSMD1 between gastric tumor and adjacent tumor free tissues, **P $<0.01$. (D) Western blot analysis of CSMDI protein in GC and paired normal tissues. Representative photos were shown in 3 paired tumor $(\mathrm{T})$ and normal $(\mathrm{N})$ tissues. The comparison of CSMD1 protein expression between IHC staining scores and western blot results. (E) Survival curves were plotted based on the Kaplan-Meier survival analysis. The expression level of CSMD1 was used as the variate to separate two lines $(P=0.016)$. 
A

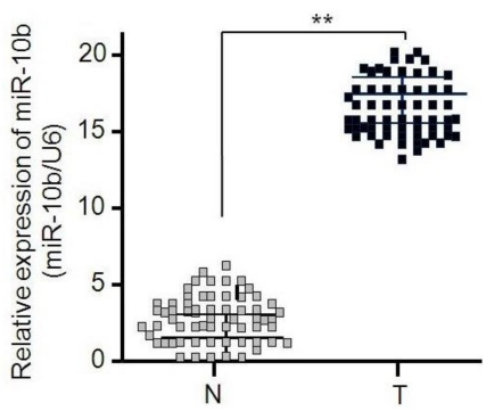

B

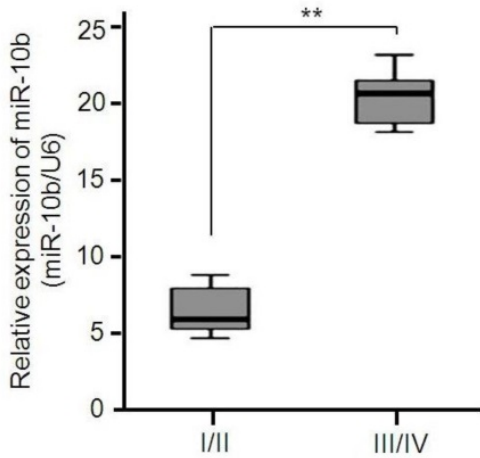

C
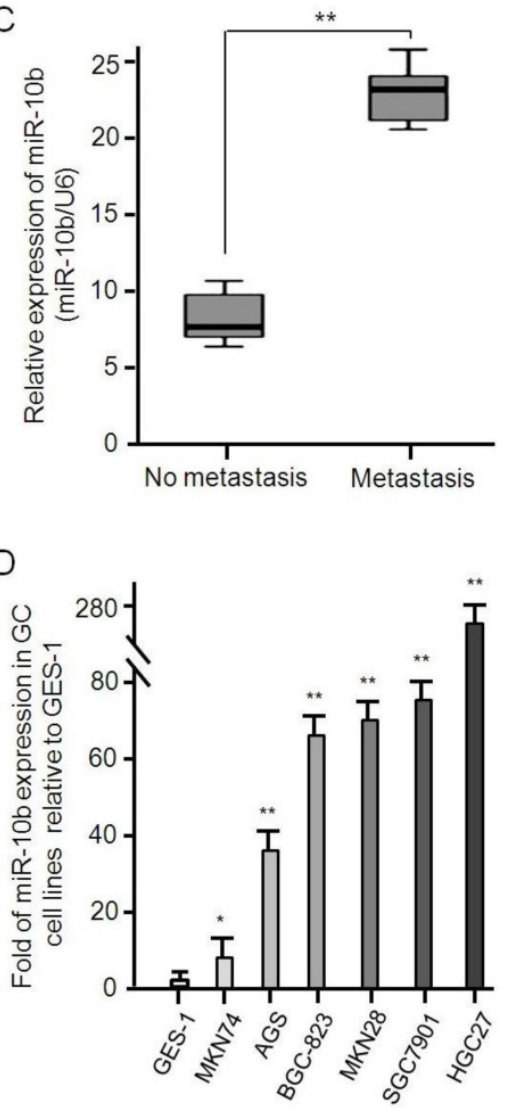

Figure 2. miR-10b is up-regulated in human GC tissues and cell lines. (A) The expression of miR-10b in tumor and matched normal tissues from 62 GC patients was analyzed by real-time PCR, $* * P<0.01$. (B) The miR-10b expression was significantly higher in patients with stage III/IV than in those with stage $1 / I I$, $* * P<0.01$. (C) The miR-10b expression was significantly higher in patients with metastasis than in those without metastasis, $* * P<0.01$. (D) The relative miR-10b expression normalized by $U 6$ in seven GC cell lines was detected by real-time RT-PCR. Data were presented as fold change in GC cell lines relative to human normal gastric epithelial cell line GES-1 and the results from three independent experiments are shown in the right as mean \pm SD. $* P<0.05, * * P<0.01$.

\section{Expressive abundance of microRNA-10b in GC tissues and cell lines}

The expressive abundance of microRNA-10b was determined in 62 pairs of GC tissues and its matched adjacent normal tissues, and U6 used to normalize the expression. As shown in Figure 2, the expression of microRNA-10b in GC tissues was significantly higher than that in adjacent normal tissues (mean \pm SD: $17.524 \pm 3.286$ vs $3.307 \pm 1.875, P<$ 0.001 ; Figure 2A). Considering TNM stage, the expressive abundance of microRNA-10b in GC tissues was positively correlated with advanced TNM stage $(P<0.001$, Figure 2B). Moreover, the expression level of microRNA-10b in GC patients with metastasis, including lymph node metastasis, was significantly higher than that in those without metastasis $(P<$ 0.001 , Figure 2C). Similarly, the expression level of microRNA-10b in GC cell lines MKN74, AGS, BGC 823, MKN28, SGC7901 and HGC27 cells was significantly higher than that in human gastric epithelial cell line GES-1 cells (Figure 2D).

\section{Oncogenic role of microRNA-10b in GC cells}

In order to evaluate the biological function of
microRNA-10b in GC cells, HGC27, a GC cell line with high expression of endogenous microRNA-10b, was stably transferred into microRNA-10b inhibitor by lentivirus infection. MTT analysis showed that the knockdown expression of microRNA-10b significantly inhibited the proliferation of HGC 27 cells in vitro (Figure 3A). Soft agar assay showed that the knockdown expression of microRNA-10b significantly inhibited the colony expansion of HGC 27 cells (Figure 3B). Then, the effects of microRNA-10b expression on migration ability of GC cells were studied by Transwell. The knockdown expression of microRNA-10b in HGC27 cells significantly repressed cell migration (Figure 3C). Also, the effect of microRNA-10b expression on the invasive properties of HGC27 cells was studied by Matrigel transmembrane invasion assay. The knockdown expression of microRNA-10b significantly reduced the invasiveness of HGC27 cells (Figure 3D). In wound healing experiment, HGC27 cells treated with miR-10b shRNA significantly reduced cell migration (Figure $3 \mathrm{E}$ and $3 \mathrm{~F}$ ). In order to investigate the potential mechanism associated with these phenotypic changes, the expressions of 
E-cadherin, Twist, Snail, Zeb1, Vimentin, NFkB-p65, iNOS, NFkB-p50, Cyclin D1, and c-Myc in these samples at the mRNA and protein levels were further detected. The results showed that the mRNA and protein levels of Twist, Snail, Zeb1, Vimentin, NFkB-p65, iNOS, NFkB-p50, Cyclin D1, and c-Myc were downregulated, while the expression level of
E-cadherin was increased, with the inhibition of microRNA-10b expression (Figure $3 \mathrm{G}$ and $3 \mathrm{H}$ ). It was suggested that microRNA-10b promotes the ability of proliferation, migration and invasion of GC cells by regulating the EMT process, and then promotes the growth and metastasis of GC.
A

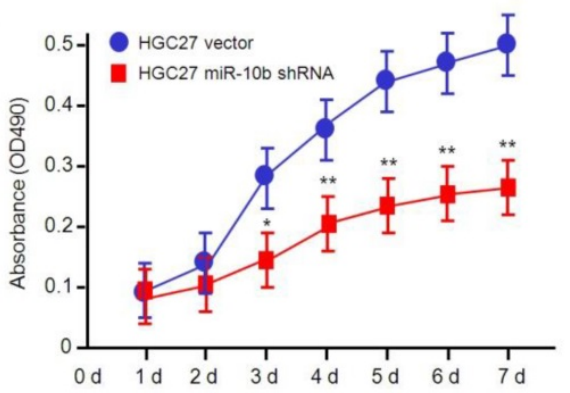

C
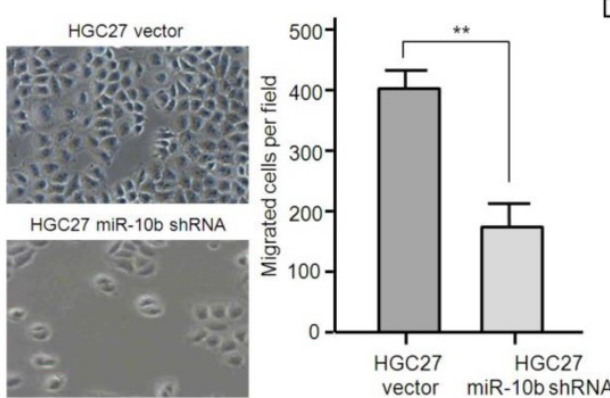

$\mathrm{E}$

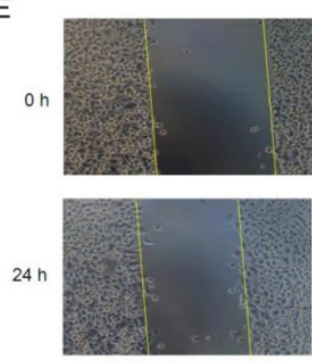

HGC27 miR-10b shRNA
B

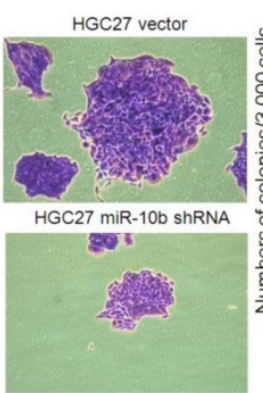

D

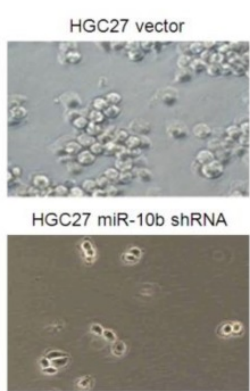

$\mathrm{F}$

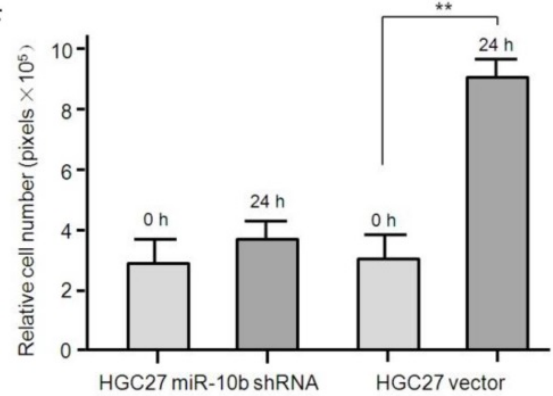

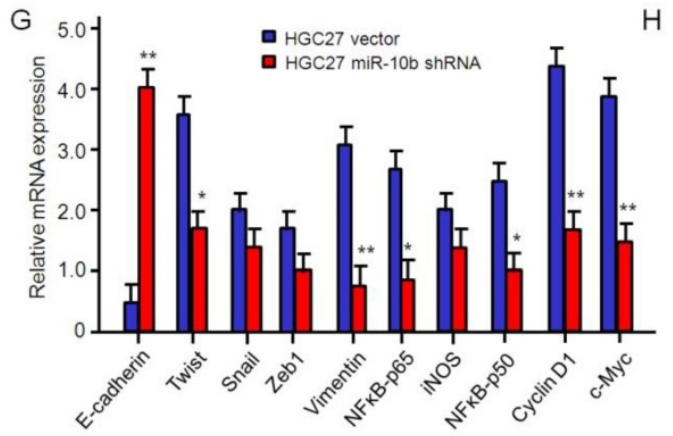

$\mathrm{H}$
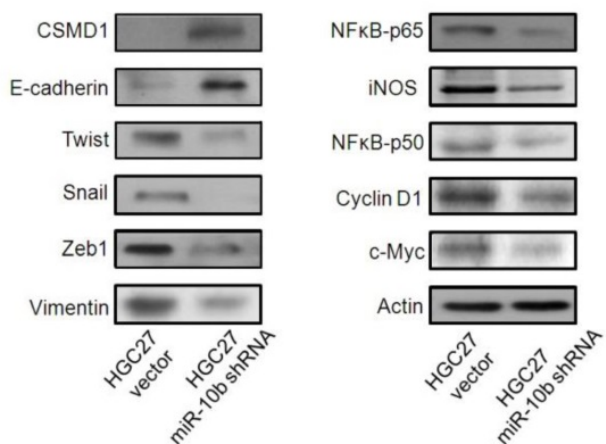

Figure 3. The knockdown of miR-10b inhibited gastric cancer cell growth, migration, and invasion in vitro. (A) The effect of miR-10b downexpression on cell proliferation in HGC27 was determined by the MTT assay. The data are calculated from triplicate experiments and shown as mean \pm SD. (B) Representative images show the colony formation of HGC27 cells with miR-10b shRNA and their control cells (left panel). Average colonies in each well for each group were counted from three independent experiments and shown as mean \pm SD (right panel). (C, D, E, F) The effects of miR-10b downexpression on cell migration, invasion and metastasis in HGC27 cells were analyzed by Transwell migration, Matrigel-coated Transwell invasion analyses and a wound healing assay. For Transwell assay and Matrigel-coated Transwell invasion analyses, the migrated cells on the bottom surface of each well were fixed and stained and the representative images are shown $(\times 100)$. The area of migrated or invading cells per field was quantified and shown in the right as mean \pm SD $(n=8) *$ and ** indicate $P<0.05$ and $P<0.01$ respectively between the corresponding groups in "HGC27 vector" vs "HGC27 miR-10b shRNA". For wound healing assay, representative images of HGC27 cells at $24 \mathrm{~h}$ after wound scratching are shown $(\times 200)$ and the results from three independent experiments are shown in the right as mean $\pm S D$. $* * P<0.01$. (G) Real-time RT-PCR analysis of EMT-and NFKB pathway-related genes. The data are calculated from triplicate experiments and shown as mean \pm SD. $* P<0.05, * * P<0.01$. (H) Western blot analysis of EMT- and NFKB pathway-related genes with the antibodies as indicated. 
A

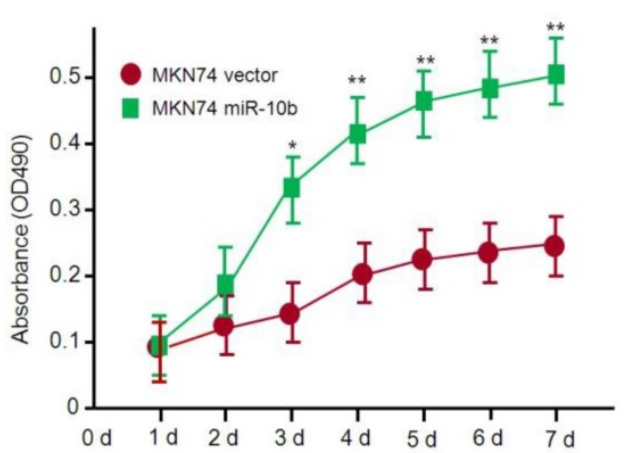

C

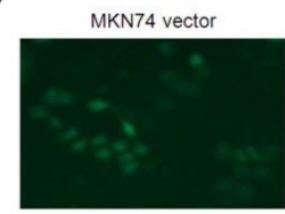

MKN74 miR-10b

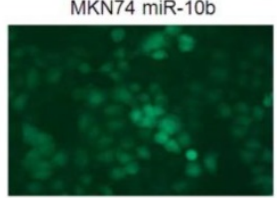

E

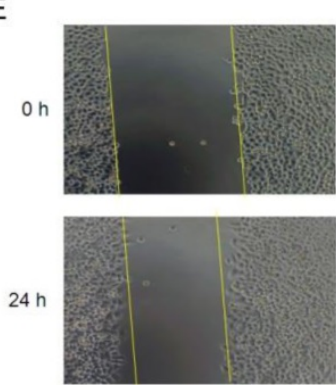

MKN74 vector

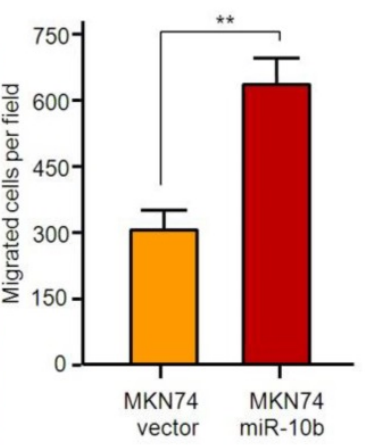

$24 \mathrm{~h}$

MKN74 miR-10b

$B$
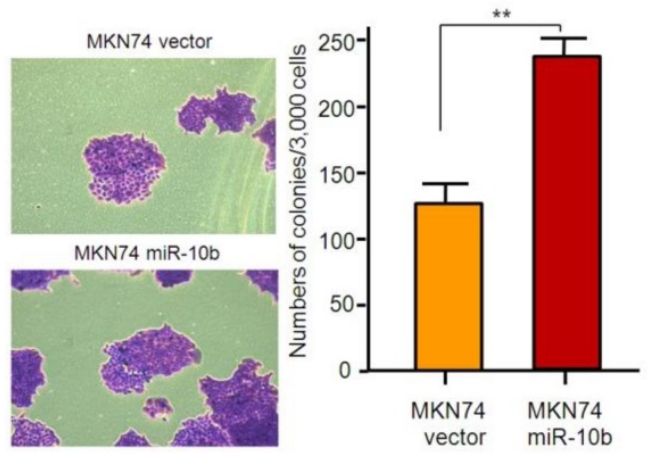

D
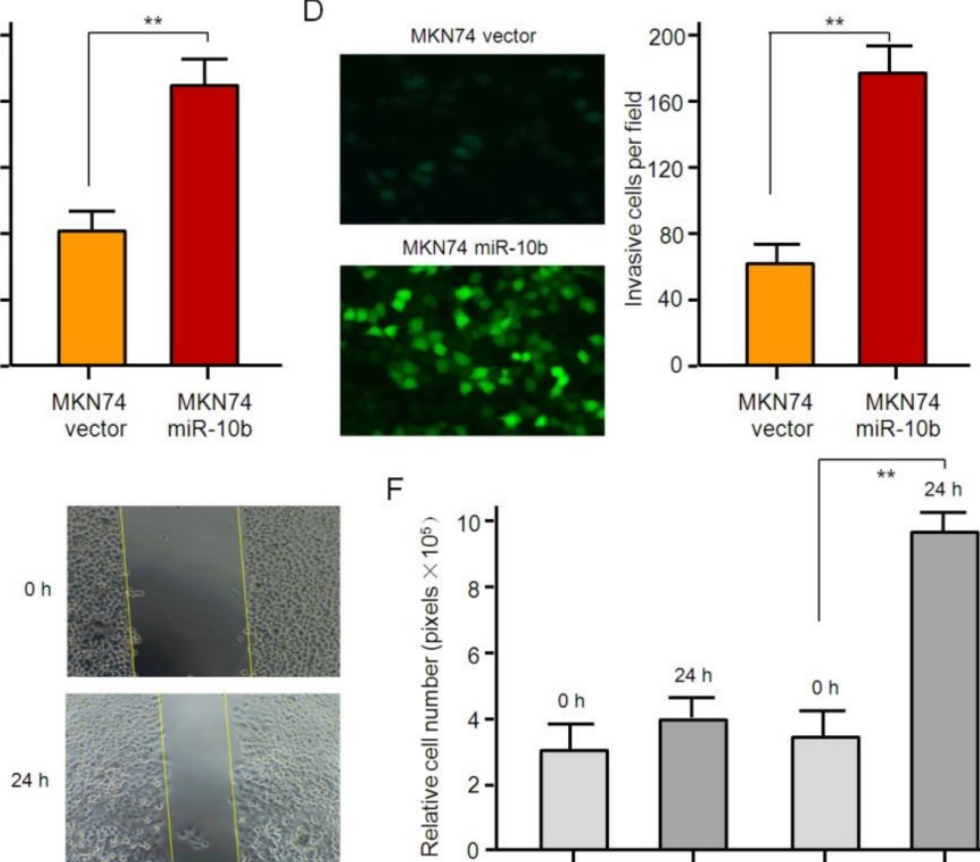

F

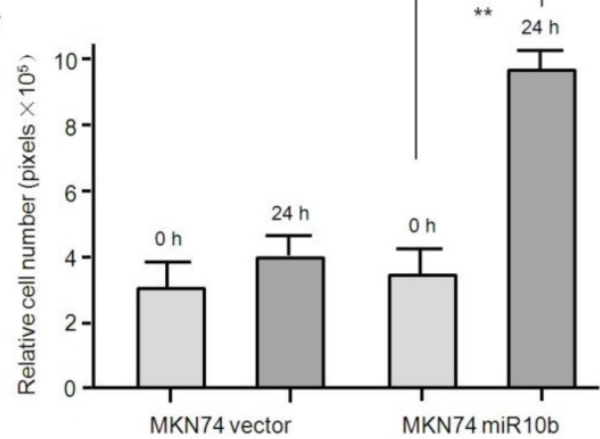

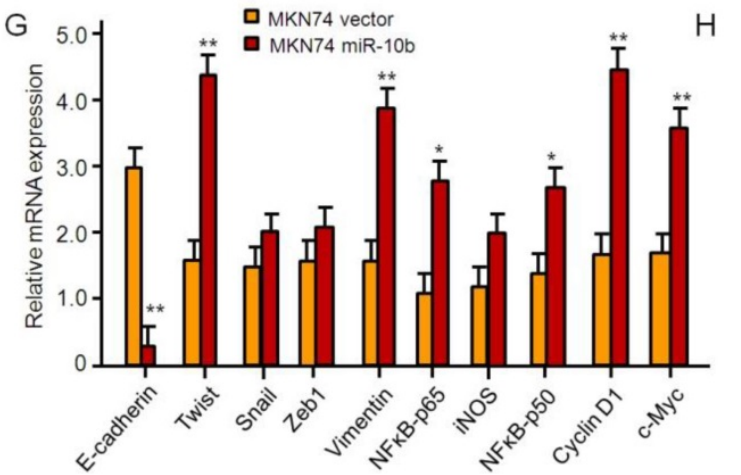
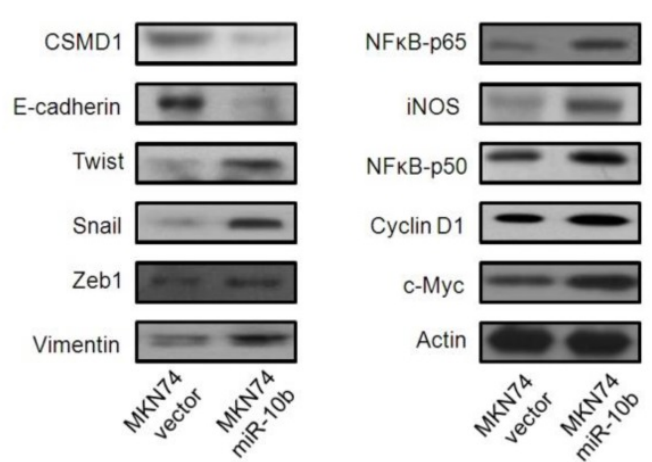

Figure 4. The overexpression of miR-1 $0 \mathrm{~b}$ promotes gastric cancer cell growth, migration, and invasion in vitro. (A) The effect of miR-10b overexpression on cell proliferation in MKN74 cells was determined by the MTT assay. The data are calculated from triplicate experiments and shown as mean \pm SD. (B) Representative images show the colony formation of MKN74 cells with miR-10b overexpression and their control cells (left panel). Average colonies in each well for each group were counted from three independent experiments and shown as mean \pm SD (right panel). (C, D, E, F) The effects of miR-10b overexpression on cell migration, invasion and metastasis in MKN74 cells were analyzed by Transwell migration, Matrigel-coated Transwell invasion analyses, with immunofluorescence staining, and a wound healing assay. For Transwell assay and Matrigel-coated Transwell invasion analyses, the migrated cells on the bottom surface of each well were fixed and stained and the representative images are shown $(\times 100)$. The area of migrated or invading cells per field was quantified and shown in the right as mean $\pm S D(n=8)$. ${ }^{*}$ and $* *$ indicate $P<0.05$ and $P<0.01$ respectively between the corresponding groups in "MKN74 vector" vs "MKN74 miR-10b". For wound healing assay, representative images of HGC27 cells at $24 \mathrm{~h}$ after wound scratching are shown $(\times 200)$ and the results from three independent experiments are shown in the right as mean \pm SD. **P $<0.01$. (G) Real-time RT-PCR analysis of EMT- and NFKB pathway-related genes. The data are calculated from triplicate experiments and shown as mean \pm SD. $* P<0.05, * * P<0.01$. (H) Western blot analysis of EMT- and NFKB pathway-related genes with the antibodies as indicated.

The ability of proliferation, invasion, and metastasis of MKN74 cells, whose endogenous
microRNA-10b was normally expressed, was strengthened by microRNA-10b overexpression with 
lentiviral infection. The results of the MTT and soft agar assay showed that the overexpression of microRNA-10b promoted the proliferation and the colony formation of MKN74 cells (Figure 4A and 4B). Similar data were obtained by Transwell analysis. The overexpression of microRNA-10b promoted invasion and migration of MKN74 cells (Figure 4C-4F). The overexpression of microRNA-10b enhanced the ability of migration and invasion of MKN74 cells (Fig. $4 \mathrm{C}-4 \mathrm{~F}$ ), up-regulated the expression of Twist, Snail, Zeb1,Vimentin, NFkB-p65, iNOS, NFkB-p50, Cyclin D1, and c-Myc at the mRNA and protein levels, while the expression of E-cadherin was significantly down-regulated (Figure $4 \mathrm{G}$ and $4 \mathrm{H}$ ).

In order to investigate the effect of down-regulation of NF-kB p65 on the cell proliferation and invasion of GC cells, a recombinant plasmid vector containing shRNA interference sequence and targeting NF-kB p65 gene was constructed and transformed into HGC 27 cells. The results showed that there was no significant difference in the proliferation ability between the pSilencer-NF- $\mathrm{kB}$ p65-shRNA transfected group and the pSilencer-Nc shRNA transfected group and the blank control group $12 \mathrm{~h}$ after transfection (both $P>0$. 05). $24 \mathrm{~h}$ after transfection, the proliferation ability of the pSilencer-NF-kB p65-shRNA transfected group was significantly lower than that of the pSilencer-NC-shRNA transfected group and the blank control group (both $P<0.05$ ). There was no significant difference in proliferation ability between the pSilencer-NC-shRNA transfected group and the blank control group $(P>0.05)$. The results showed that the proliferation activity of HGC27 cells was inhibited after decreasing the expression of NF- $\mathrm{kB}$ p65, that is, NF-kB p65 could promote the proliferation of GC cells. The results of the Transwell chamber invasion assay demonstrated that the number of perforated cells in the pSilencer-NF-kB p65-shRNA transfection group was $35.8 \pm 5.4$. Compared with the pSilencer-NC-shRNA transfection group (68.2 \pm 7.6$)$ and blank control group (65.6 \pm 7.2$)$, the difference was statistically significant (both $P<0$. 05). But, there was no significant difference in the number of transmembrane cells between the pSilencer-NCshRNA transfection group and the blank control group $(P>0.05)$. These results indicated that NF-kB p65 could promote the invasion ability of HGC27 cells.

\section{microRNA-10b directly targeted CSMDI in GC cells}

It has been reported that CSMD1 is regulated by microRNA-10b in human hepatocellular carcinoma cells [45]. The Luciferase reporting assays were carried out to determine whether microRNA-10b was directly targeted CSMD1 in GC cells. The microRNA-10b binding site 707-713 was identified in the CSMD1 3'-UTR. pMIR-CSMD1-3'-UTR-WT contained the 707-713 binding sites. pMIR-CSMD1-3'UTR-mut contained a mutation in the 707-713 (TGTCCCA) site. Therefore, a luciferase reporter assay was performed to confirm the binding ability of microRNA-10b to CSMD1 cDNA. It was found that the overexpression of microRNA-10b significantly inhibited the expression of luciferase in HGC27 cells transfected with pMIR-CSMD1-3'-UTR-WT but not with pMIR-CSMD1-3'-UTR-mut (Figure 5A). The luciferase activity of HGC 27 cells co-transfected with pMIR-CSMD1-3'-UTR-WT and pcDNA3.1microRNA-10b was 0.38 -fold lower than that of the negative control group $(P<0.05)$. A similar effect was also observed in SGC7901 cells (a 0.41-fold decrease compared with the blank control, $P<0.05$; Figure 5B). These results suggest that microRNA- $10 \mathrm{~b}$ binds to the 707-713 site of CSMD 13'-UTR in GC cells.

\section{microRNA-10b promoted growth and metastasis of gastric cancer in vivo}

In order to determine whether microRNA-10b can promote the growth and metastasis of GC cells in vivo, two groups of GC models, such as HGC27 cells with microRNA-10b shRNA and control group cells, MKN74 cells with the overexpression of microRNA-10b and control group cells thereof, were established. Then, these cells were injected subcutaneously into the nude mice. After injection of a combination of HGC27 cells with microRNA-10b shRNA for 6 weeks, the tumor volume and weight of mice were smaller and lighter than those of the mice injected with HGC27 vector cells (Figure 6A, 6B and $6 \mathrm{C})$. In addition, the mice in the control group showed obvious primary tumor, while the tumor volume and weight of the mice with combined injection of MKN74 cells and microRNA-10b overexpression was significantly increased (Figure 6D, 6E and 6F). By comparing the data of microRNA-10b overexpression group or microRNA-10b knockdown group and their control group at different experimental points, it was concluded that microRNA-10b might result in an average increase of the tumor growth. Only orthotopic tumors were found in the HGC27 miR-10b shRNA and MKN74 vector groups, whereas tumor metastasis was found in $66.7 \%(2 / 3)$ of the MKN74 miR-10b-overexpressed group and $33.3 \%(1 / 3)$ of the HGC27 vector group. These results suggested that miR-10b promoted the metastasis of GC cells. 
A

\begin{tabular}{lllll} 
pCDNA3.1 & + & - & + & - \\
pCDNA3.1-miR-10b & - & + & - & + \\
pMIR-CSMD1-3'-UTR-WT & + & + & - & - \\
pMIR-CSMD1-3'-UTR-mut & - & - & + & + \\
\hline
\end{tabular}

HGC27
B

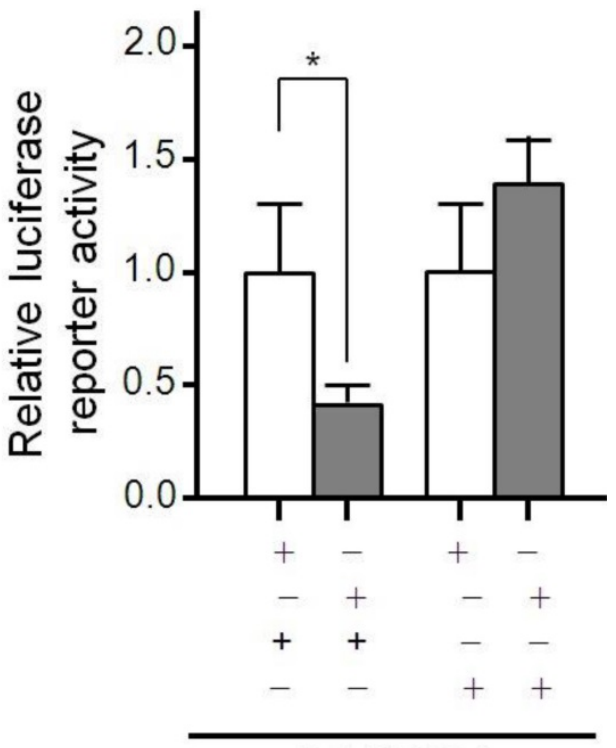

SGC7901

Figure 5. The CSMD1-3'-UTR is a target of miR-10b. The diagram of the luciferase reporter plasmids: plasmid with the full length wild-type CSMDI-3'-UTR (pMIR- CSMD1-3'-UTR-WT) insert and plasmid with a mutant CSMD1-3'-UTR (PMIR-CSMD1-3'-UTR-mut) which carried a substitution of seven nucleotides (TGTCCCA) within the miR-10b binding site (707-713). Luciferase activity assay demonstrates a direct targeting of the 3'-UTR of CSMD1 by miR-10b. HGC27 and SGC7901 cells were transfected with pcDNA3.1-miR-10b and pMIR-CDMD1-3'- UTR-WT or PMIR-CSMD1-3'-UTR-mut. (A) HGC27 cells; (B) SGC7901 cells.

A

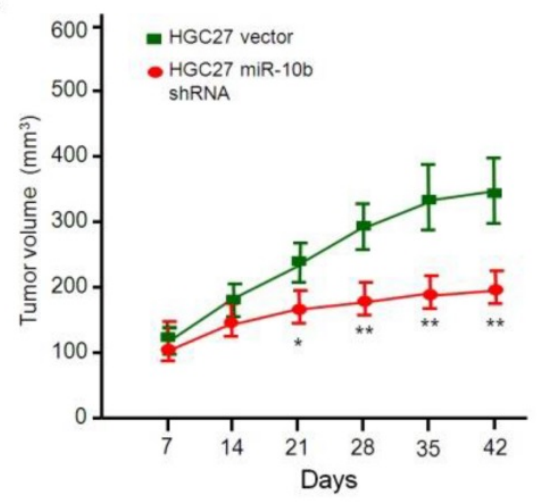

D

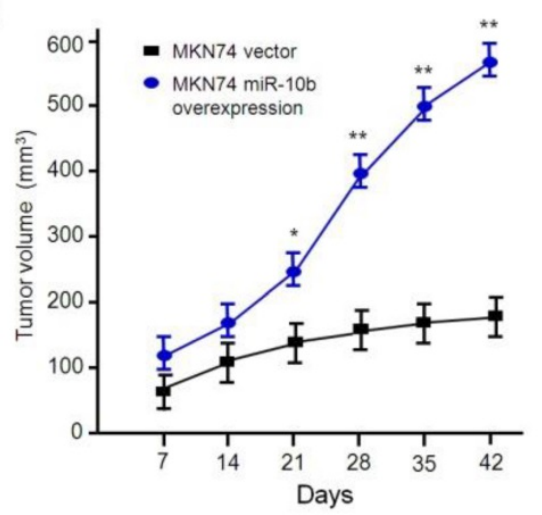

B

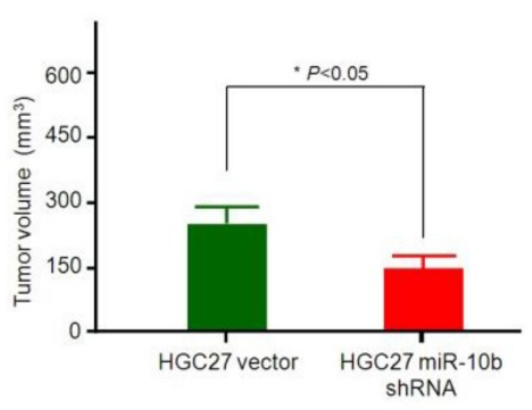

E

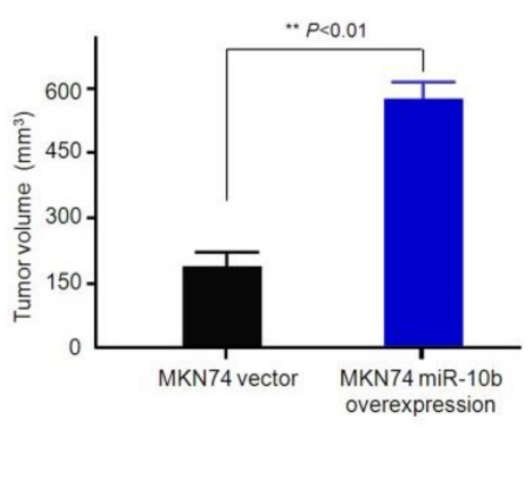

C

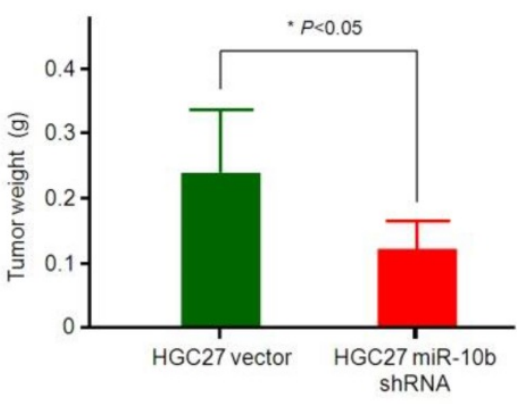

$\mathrm{F}$

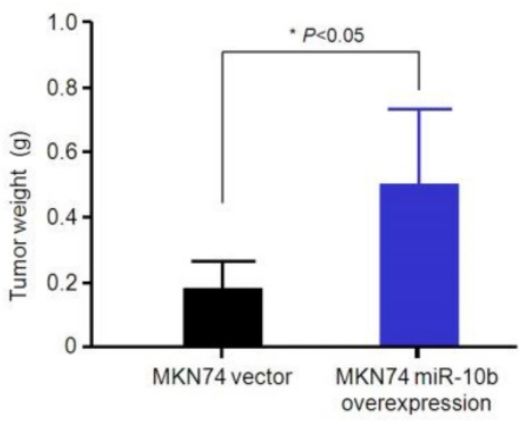

Figure 6. Effect of miR-10b expression on GC growth in vivo. (A, D) Determination of tumor volumes at different time points in (A) HGC27 miR-10b shRNA vs HGC27 vector, (D) MKN74 miR-10b overexpression vs MKN74 vector. The data are calculated from 3 nude mice per group and shown as mean \pm SD. $* P<0.05$, $* * P<0.01$. (B, E) After the final measure, the mice were sacrificed, and the tumors were excised. Tumor volume was measured and calculated using the formula length $\times$ width $2 / 2$. Student's $t$ test was used to analyze the significant differences. The data are calculated from 3 nude mice per group and shown as mean \pm SD. $* P<0.05$, $* * P<0.01$. (B) HGC27 miR-10b shRNA vs HGC27 vector, (E) MKN74 miR-10b overexpression vs MKN74 vector. (C, F) After the final measure, the mice were sacrificed, and the tumors were excised. Tumor weight was measured and the Student's $t$ test was used to analyze the significant differences. The data are calculated from 3 nude mice per group and shown as mean \pm SD. $* P<0.05$, $* * P<$ 0.01 . (C) HGC27 miR-10b shRNA vs HGC27 vector, (F) MKN74 miR-10b overexpression vs MKN74 vector. 


\section{Discussion}

Metastasis is a complex multistep process triggered by a body of transcriptive factors. microRNA plays an important role in regulating gene expression [20-22]. Aberrant microRNA expression level in various human cancers contributes to tumor progression at different stages by inhibiting their target genes [46]. Therefore, the recognition of specific microRNA and its targets related to tumorigenesis and metastasis can provide clues for tumor diagnosis, treatment and prevention. microRNA-10b is located in the HOX gene cluster on chromosome 2, which is closely related to tumor invasion and metastasis. Previous studies have shown that microRNA-10b is overexpressed in breast cancer [23], colorectal cancer [24], head and neck cancer [25], pancreatic cancer [26], glioblastoma [27], nasopharyngeal carcinoma [28], hepatocellular carcinoma [29] and so on. Consistent with previous reports, this study showed that the expression of microRNA-10b in GC tissues and 6 GC cell lines was higher than that in paracancerous non-tumor tissues and GES-1 cell line. Besides, the expression of microRNA-10b in patients with metastasis, including lymph node metastasis, was significantly higher than that in patients without metastasis. The up-regulation of microRNA-10b has been shown to promote the invasion and metastasis of various tumors [23-29]. In this study, the knockdown of miR-10b expression inhibited the growth, migration and invasion of HGC27 cells. The converse was also true. Overexpression of microRNA-10b promoted the growth, migration and invasion of MKN74 cells. Taken together, these results were consistent with the previous findings, showing that microRNA-10b promoted the metastasis of GC [38-41], suggesting that microRNA-10b exerted oncogenic activity in GC cells, however, the mechanism by which it drives tumor metastasis is unclear. Previous studies have revealed that microRNA-10b promotes the metastasis of a variety of tumor cells by regulating Bim, TFAP2C, P16, P21, E-cadherin, CD138, RHOC, RhoC, UPAR, MMP-2, MMP-9, HOXD10, etc [23-29]. In this study, microRNA-10b was involved in the proliferation and metastasis of GC cells by targeting CSMD1, and facilitated epithelial-mesenchyma transformation (EMT) process by the NF-kB pathway.

CSMD1 is localized on chromosome 8p23.2, which is a putative tumor suppressor gene [4]. Mounting evidence indicates that the deletion of 8p23.2 or reduced expression of CSMD1 is associated with the development of many cancers [5-9]. Multiple mechanisms can reduce the expression of CSMD1. The allelic loss, mutation and methylation of CSMD1 have been detected in breast cancer, head and neck cancer, oral squamous cell carcinoma, prostate cancer, rectum, liver, lung and skin cancer and many other malignant tumors such as breast cancer, head and neck cancer, oral squamous cell carcinoma, prostate, colorectal cancer, liver cancer, lung cancer and skin cancer, etc [5,6,8-16]. Using four databases of TargetScan, Miranda, miRWalk and PicTar, highly conserved microRNA-10b binding sites were found in CSMD1 3'-UTRs. In our study, the molecular mechanism of the correlation between miR-10b and CSMD1 was further was further investigated. The results revealed that miR- $10 \mathrm{~b}$ could bind to the 707-713 binding sites in the 3'-UTRs of CSMD1. It was found that the expression of microRNA-10b in GC cells was negatively correlated with the expression of CSMD1, suggesting that microRNA-10b could promote the proliferation and metastasis of GC cells at least partly by down-regulating the expression of CSMD1.

In a word, the expression of microRNA-10b in GC tissues and cell lines were analyzed, and its effect on cells was studied. It was revealed that the overexpression of microRNA- $10 \mathrm{~b}$ enhanced the vitality, migration and invasion of GC cells. The study provided evidence that CSMD1 is indeed a direct target of microRNA-10b in GC cells, and microRNA-10b could mediate an oncogenic effect in GC by targeting CSMD1 expression. In addition, this study also provided potential diagnostic and prognostic markers and promising candidates for effective GC therapeutic strategies.

\section{Supplementary Material}

Supplementary figures and tables. http://www.ijbs.com/v15p2075s1.pdf

\section{Acknowledgments}

This research was partly supported by a grant from the National Health and Family Planning Commission Scientific Research Foundation-Zhejiang Medical and Health Major Science and Technology Plan (WKJ-ZJ-1505), Major Program of Science and Technology of Zhejiang Province (2013C03044-4), the Leading Talents in Scientific and Technological Innovation from Zhejiang Provincial Ten Thousand Talents Plan (Zhejiang Provincial CPC Committee Talents [2019]-3), Zhejiang Provincial Program for the Cultivation of High-level Innovative Health talents (zjwjw2014-108), and the Major Training Personnel from Zhejiang Provincial Program for the Training and Development Project for 151 talents (zjhrss2014-150). 


\section{Competing Interests}

\section{This study is not related to any potentially competing financial or other interests.}

\section{References}

1. Rawla P, Barsouk A. Epidemiology of gastric cancer: global trends, risk factors and prevention. Prz Gastroenterol. 2019; 14:26-38

2. Varga MG, Cai H, Waterboer T, et al. Epstein-Barr Virus Antibody Titers Are Not Associated with Gastric Cancer Risk in East Asia. Dig Dis Sci. 2018; 63: 2765- 2772.

3. Wang FH, Shen L, Li J, et al. The Chinese Society of Clinical Oncology (CSCO): clinical guidelines for the diagnosis and treatment of gastric cancer. Cancer Commun (Lond). 2019; 39:10.

4. Gialeli C, Gungor B, Blom AM. Novel potential inhibitors of complement system and their roles in complement regulation and beyond. Mol Immunol. 2018; 102:73-83.

5. Jung AR, Eun YG, Lee YC, et al. Clinical Significance of CUB and Sushi Multiple Domains 1 Inactivation in Head and Neck Squamous Cell Carcinoma. Int J Mol Sci. 2018; 19(pii: E):3996

6. Lee $\mathrm{JH}, \mathrm{An} \mathrm{CH}$, Kim MS, et al. Rare frameshift mutations of putative tumor suppressor genes CSMD1 and SLX4 in colorectal cancers. Pathol Res Pract. 2018; 214:325-326

7. Escudero-Esparza A, Bartoschek M, Gialeli C, et al. Complement inhibitor CSMD1 acts as tumor suppressor in human breast cancer. Oncotarget. 2016; 7:76920-76933.

8. Zhu Q, Gong L, Wang J, et al. miR-10b exerts oncogenic activity in human hepatocellular carcinoma cells by targeting expression of CUB and sushi multiple domains 1 (CSMD1). BMC Cancer. 2016; 16:806.

9. Zhang R, Song C. Loss of CSMD1 or 2 may contribute to the poor prognosis of colorectal cancer patients. Tumour Biol. 2014; 35:4419-4423.

10. Kayser K, Degenhardt F, Holzapfel S, et al. Copy number variation analysis and targeted NGS in 77 families with suspected Lynch syndrome reveals novel potential causative genes. Int J Cancer. 2018; 143:2800-2813.

11. Gong S, Johnson MD, Dopierala J, et al. Genome-wide oxidative bisulfite sequencing identifies sex-specific methylation differences in the human placenta. Epigenetics. 2018; 13:228-239.

12. Kamal M, Holliday DL, Morrison EE, et al. Loss of CSMD1 expression disrupts mammary duct formation while enhancing proliferation, migration and invasion. Oncol Rep. 2017; 38:283-292.

13. Escudero-Esparza A, Bartoschek M, Gialeli C, et al. Complement inhibitor CSMD1 acts as tumor suppressor in human breast cancer. Oncotarget. 2016; 7: 76920-76933.

14. Zhu Q, Gong L, Liu X, et al. Loss of heterozygosity at D8S262: an early genetic event of hepatocarcinogenesis. Diagn Pathol. 2015; 10:70.

15. Ali Hassan NZ, Mokhtar NM, Kok Sin T, et al. Integrated analysis of copy number variation and genome-wide expression profiling in colorectal cancer tissues. PLoS One. 2014; 9:e92553.

16. Shull AY, Clendenning ML, Ghoshal-Gupta S. et al. Somatic mutations, allele loss, and DNA methylation of the Cub and Sushi Multiple Domains 1 (CSMD1) gene reveals association with early age of diagnosis in colorectal cancer patients. PloS one. 2013; 8:e58731.

17. Bosisio D, Gianello V, Salvi V, et al. Extracellular miRNAs as activators of innate immune receptors. Cancer Lett. 2019; 452:59-65.

18. Schwarzenbach H, Gahan PB. MicroRNA Shuttle from Cell-To-Cell by Exosomes and Its Impact in Cancer. Noncoding RNA. 2019; 5(pii: E):28.

19. Yao $Q$, Chen $Y$, Zhou $X$. The roles of microRNAs in epigenetic regulation. Curr Opin Chem Biol. 2019; 51:11-17.

20. Lee SS, Cheah YK. The Interplay between MicroRNAs and Cellular Components of Tumour Microenvironment (TME) on Non-Small-Cell Lung Cancer (NSCLC) Progression. J Immunol Res. 2019; 2019:3046379.

21. Yang CX, Sedhom W, Song J, et al. The Role of MicroRNAs in Recurrence and Metastasis of Head and Neck Squamous Cell Carcinoma. Cancers (Basel). 2019; 11(pii: E):395

22. Alečković M, Kang Y. Regulation of cancer metastasis by cell-free miRNAs. Biochim Biophys Acta. 2015; 1855:24-42.

23. Khalighfard S, Alizadeh AM, Irani S, et al. Plasma miR-21, miR-155, miR-10b, and Let-7a as the potential biomarkers for the monitoring of breast cancer patients. Sci Rep. 2018; 8:17981.

24. Jiang H, Liu J, Chen Y. et al. Up-regulation of mir-10b predicate advanced clinicopathological features and liver metastasis in colorectal cancer. Cancer Med. 2016; 5:2932-2941.

25. Bourguignon LYW, Earle C, Shiina M. Activation of Matrix Hyaluronan-Mediated CD44 Signaling, Epigenetic Regulation and Chemoresistance in Head and Neck Cancer Stem Cells. Int J Mol Sci. 2017; 18(pii: E):1849.

26. Lai X, Wang M, McElyea SD, et al. A microRNA signature in circulating exosomes is superior to exosomal glypican-1 levels for diagnosing pancreatic cancer. Cancer Lett. 2017; 393:86-93.

27. El Fatimy R, Subramanian S, Uhlmann EJ, et al. Genome Editing Reveals Glioblastoma Addiction to MicroRNA-10b. Mol Ther. 2017; 25:368-378.
28. Zhang $\mathrm{P}$, Hong $\mathrm{H}$, Sun $\mathrm{X}$ et al. MicroRNA-10b regulates epithelial-mesenchymal transition by modulating KLF4/Notch1/E-cadherin in cisplatin-resistant nasopharyngeal carcinoma cells. Am J Cancer Res. 2016; 6:141-156

29. Liao CG, Kong LM, Zhou P. et al. miR-10b is overexpressed in hepatocellular carcinoma and promotes cell proliferation, migration and invasion through RhoC, uPAR and MMPs. J Transl Med. 2014; 12:234.

30. Liang HX, Sun LB, Liu NJ. Neferine inhibits proliferation, migration and invasion of U251 glioma cells by down-regulation of miR-10b. Biomed Pharmacother. 2019; 109:1032-1040.

31. Sheedy $P$, Medarova $Z$. The fundamental role of miR-10b in metastatic cancer. Am J Cancer Res. 2018; 8:1674-1688.

32. Yang J, Wang S, Wang F. et al. Downregulation of miR-10b promotes osteoblast differentiation through targeting Bcl6. Int I Mol Med. 2017; 39:1605-1612.

33. Wang J, Wang B, Chen LQ. et al. miR-10b promotes invasion by targeting KLF4 in osteosarcoma cells. Biomed Pharmacother. 2016; 84:947-953.

34. Ma C, Wei F, Xia H. et al. MicroRNA-10b mediates TGF- $\beta 1$-regulated glioblastoma proliferation, migration and epithelial-mesenchymal transition. Int J Oncol. 2017; 50:1739-1748.

35. Khella HWZ, Daniel N, Youssef L et al. miR-10b is a prognostic marker in clear cell renal cell carcinoma. J Clin Pathol. 2017; 70:854-859.

36. Zhang Y, Wang LJ, Yang HQ, et al. MicroRNA-10b expression predicts long-term survival in patients with solid tumor. J Cell Physiol. 2019; 234:1248-1256.

37. Huang $\mathrm{Q}$, Song $\mathrm{Q}$, Zhong $\mathrm{W}$, et al. MicroRNA-10b and the clinical outcomes of various cancers: A systematic review and meta-analysis. Clin Chim Acta. 2017; 474:14-22.

38. Kim K, Lee HC, Park JL. et al. Epigenetic regulation of microRNA-10b and targeting of oncogenic MAPRE1 in gastric cancer. Epigenetics. 2011; 6:740-751.

39. Li Z, Lei H, Luo M. et al. DNA methylation downregulated mir-10b acts as a tumor suppressor in gastric cancer. Gastric Cancer. 2015; 8:43-54.

40. Liu Z, Zhu J, Cao H. et al. miR-10b promotes cell invasion through RhoC-AKT signaling pathway by targeting HOXD10 in gastric cancer. Int J Oncol. 2012; 40:1553-1560.

41. Wang YY, Ye ZY, Zhao ZS. et al. Clinicopathologic significance of miR-10b expression in gastric carcinoma. Hum Pathol. 2013; 44:1278-1285.

42. Ling ZQ, Guo W, Lu XX. et al. A Golgi-specific protein PAQR3 is closely associated with the progression, metastasis and prognosis of human gastric cancers. Ann Oncol. 2014; 25:1363-1372.

43. Ling ZQ, Lv P, Lu XX. et al. Circulating Methylated XAF1 DNA Indicates Poor Prognosis for Gastric Cancer. PLoS One. 2013; 8:e67195.

44. Sun L, Yan W, Wang Y. et al. MicroRNA-10b induces glioma cell invasion by modulating MMP-14 and uPAR expression via HOXD10. Brain Res. 2011; 1389:

45. Zhu Q, Gong L, Wang J. et al. miR-10b exerts oncogenic activity in human hepatocellular carcinoma cells by targeting expression of CUB and sushi multiple domains 1 (CSMD1). BMC Cancer. 2016; 16:806.

46. Liu B, Shyr Y, Cai J, et al. Interplay between miRNAs and host genes and their role in cancer. Brief Funct Genomics. 2019; pii: elz002. 